TRANSACTIONS OF THE

AMERICAN MATHEMATICAL SOCIETY

Volume 357, Number 9, Pages 3725-3738

S 0002-9947(05)03675-5

Article electronically published on March 25, 2005

\title{
COMPLEX IMMERSIONS IN KÄHLER MANIFOLDS OF POSITIVE HOLOMORPHIC $k$-RICCI CURVATURE
}

\author{
FUQUAN FANG AND SÉRGIO MENDONÇA
}

\begin{abstract}
The main purpose of this paper is to prove several connectedness theorems for complex immersions of closed manifolds in Kähler manifolds with positive holomorphic $k$-Ricci curvature. In particular this generalizes the classical Lefschetz hyperplane section theorem for projective varieties. As an immediate geometric application we prove that a complex immersion of an $n$ dimensional closed manifold in a simply connected closed Kähler $m$-manifold $M$ with positive holomorphic $k$-Ricci curvature is an embedding, provided that $2 n \geq m+k$. This assertion for $k=1$ follows from the Fulton-Hansen theorem (1979).
\end{abstract}

\section{INTRODUCTION}

The Lefschetz hyperplane section theorem [Le] (cf. $[\mathrm{AF}]$ ) describes how the topology of a projective algebraic manifold $X$ is related to the topology of a (generic) hyperplane section $X_{0}=X \cap H$, i.e. the relative homology groups

$$
H_{i}\left(X, X_{0}\right)=0
$$

for $i \leq n-1$, where $H$ is a generic hyperplane and $n$ is the dimension of $X$.

Fifty years later Barth ([Ba] $)$ generalized Lefschetz's theorem to the intersection of any two projective algebraic manifolds $X \subset \mathbb{P}^{v}$ and $Y \subset \mathbb{P}^{v}$, asserting that

$$
H_{i}(X, X \cap Y)=0
$$

for $j \leq \min (n+m-v, 2 m-v+1)$, where $n=\operatorname{dim} X$ and $m=\operatorname{dim} Y$.

Various generalizations of both results, e.g. to homotopy groups, to local complete intersections and to singular varieties, have been a theme in projective algebraic geometry, and were pioneered in recent times by W. Fulton and several other mathematicians, especially P. Deligne, G. Faltings, T. Gaffney, M. Goresky, K. Johnson, J.P. Jouanolou, R. Lazarsfeld, R. MacPherson, J. Roberts and F.L. Zak (cf. $[\mathrm{GM}],[\mathrm{FL}]$ and $[\mathrm{Fu}]$ for a more complete survey and references therein).

The well-known Mori-Siu-Yau theorem which solved the Frankel conjecture (cf. $[\mathrm{Fr},[\mathrm{SY}], \mathrm{MO}]$ ) shows that compact Kähler manifolds of positive holomorphic bisectional curvature are bi-holomorphic to $\mathbb{P}^{m}$. Theorems of Barth-Lefschetz type for embedded complex submanifolds in Kähler manifolds with positive holomorphic bisectional curvature are proved in $\underline{\mathrm{SW}}$ by Schoen and Wolfson using Morse theory on path spaces. In particular, it gives proofs of the Barth and Lefschetz theorems

Received by the editors August 5, 2003 and, in revised form, March 10, 2004.

2000 Mathematics Subject Classification. Primary 32Q15; Secondary 53C55.

The first author was supported by NSFC Grant 19741002, RFDP and the Qiu-Shi Foundation. 
using differential geometry methods. In [SW], and very recently [KW], BarthLefschetz type theorems on Kähler manifolds of nonnegative bisectional curvature are further studied. A key numerical invariant, called complex positivity, is involved.

The main purpose of this paper is to prove several more general connectedness theorems for immersed complex submanifolds in complete Kähler manifolds of positive holomorphic $k$-Ricci curvature (defined below, compare [Sh], [Wu] $)$, which coincides with the holomorphic bisectional curvature if $k=1$. For Kähler manifolds of nonnegative bisectional curvature, the maximal number $k$ for which the holomorphic $k$-Ricci curvature is positive, is closely related to the complex positivity. In particular, our results imply connectedness theorems for Kähler homogenous spaces (cf. [KW], $\mathrm{Ok},[\mathrm{So}$ ). As a geometric application we prove a generalized regularity theorem of Fulton-Hansen type (cf. [FH]): Let $f: N \rightarrow M$ be a complex immersion of an $n$-dimensional closed manifold $N$ into a complete simply connected Kähler manifold $M$ of dimension $m$. If the holomorphic $k$-Ricci curvature of $M$ is positive, then $f$ is an embedding, provided that $2 n \geq m+k$.

To obtain the above-mentioned regularity theorem (cf. Theorem 0.2 below), our starting point is to adapt the well-known Morse theory to a certain twisted path space associated to the immersion, instead of embeddings (compare [SW]).

We begin with the definition of holomorphic $k$-Ricci curvature.

Let $M$ be an $m$-dimensional Kähler manifold with complex structure $J$. Given pairwisely orthogonal complex lines, $\sigma_{0}, \sigma_{1}, \cdots, \sigma_{k}$, we define the holomorphic $k$ Ricci curvature associated to $\sigma_{0}, \sigma_{1}, \cdots, \sigma_{k}$ to be

$$
\operatorname{Ric}_{k}^{H}\left(\sigma_{0}, \sigma_{1}, \cdots, \sigma_{k}\right):=\sum_{j=1}^{k} H\left(\sigma_{0}, \sigma_{j}\right),
$$

where $H\left(\sigma_{0}, \sigma_{j}\right)$ is the holomorphic sectional curvature of $\left(\sigma_{0}, \sigma_{j}\right)$. We say the holomorphic $k$-Ricci curvature of $M$ is positive if $\operatorname{Ric}_{k}^{H}\left(\sigma_{0}, \sigma_{1}, \cdots, \sigma_{k}\right)>0$ for any pairwisely orthogonal complex lines, $\sigma_{0}, \sigma_{1}, \cdots, \sigma_{k}$. Recall that $H\left(\sigma_{0}, \sigma_{j}\right)=$ $\langle R(X, J X), J Y, Y\rangle$, where $X \in \sigma_{0}$ and $Y \in \sigma_{j}$ are unit vectors (cf. [GK]).

For the sake of simplicity manifolds, denoted by $M, N, N_{i}$ in the paper, are all closed and connected.

Theorem A. Let $M$ be a complete $m$-dimensional Kähler manifold with positive holomorphic $k$-Ricci curvature. Let $\Delta$ be the diagonal of $M \times M$. If $f: N \rightarrow M \times M$ is a complex immersion where $N$ is a closed complex manifold of dimension $n$, then:

(A1) If $n>m+k-1$, then $f^{-1}(\Delta)$ is nonempty.

(A2) If $n>m+k$ and $M$ is simply connected, then $f^{-1}(\Delta)$ is connected.

(A3) If $i<n-m-k+1$ there is an exact sequence of homotopy groups

$$
\pi_{i}\left(f^{-1}(\Delta)\right) \rightarrow \pi_{i}(N) \stackrel{\left(p_{1} f\right)_{*}-\left(p_{2} f\right)_{*}}{\longrightarrow} \pi_{i}(M) \rightarrow \pi_{i-1}\left(f^{-1}(\Delta)\right) \rightarrow \cdots,
$$

where $p_{1}, p_{2}$ are the projections of $M \times M$ to the first and second factors, respectively.

From the definition it is obvious that holomorphic 1-Ricci curvature is the bisectional curvature. Therefore by [SY] and [Mo] we have $M=\mathbb{P}^{m}$ if $k=1$ and $M$ is compact. In this case the above theorem reduces to the connectedness theorems of Fulton-Hansen (cf. $[\mathrm{FH}]$ ) and Deligne (cf. [GM], page 27) for local complete intersections in $\mathbb{P}^{m}$.

One may wonder if the above statements can be improved by changing the strict inequalities to weak inequalities. This may not be possible in the most general 
situation. For instance, if $k=1$ and $M=\mathbb{P}^{m}$ with $m$ odd, for a free holomorphic bijection $\phi$, the map $f: \mathbb{P}^{m} \rightarrow \mathbb{P}^{m} \times \mathbb{P}^{m}$ defined by $f(x)=(x, \phi(x))$ satisfies $f^{-1}(\Delta)=\emptyset$; compare to $(\mathrm{A} 1)$.

However, below, in the most interesting case, Theorem A is sharpened with all strict inequalities replaced by weak inequalities. Moreover, there is an additional property (see B4).

For a map $f: Y \rightarrow X$, let $X_{f}=X \cup_{f}(Y \times[0,1])$ denote the mapping cylinder of $f$. We will use $\pi_{i}(X, Y)$ to denote the relative homotopy group $\pi_{i}\left(X_{f}, Y\right)$. In the following the relative homotopy group should be understood in this way.

Theorem B. Let $M$ be a closed Kähler m-manifold of positive holomorphic $k$ Ricci curvature. Assume $f=\left(f_{1}, f_{2}\right): N \rightarrow M \times M$, where $N=N_{1} \times N_{2}$ and $f_{i}: N_{i} \rightarrow M$ are complex immersions of closed complex manifolds. Then:

(B1) If $n \geq m+k-1$, then $f^{-1}(\Delta)$ is nonempty.

(B2) If $n \geq m+k$ and $M$ is simply connected, then $f^{-1}(\Delta)$ is connected.

(B3) For $i \leq n-m-k+1$, there is an exact sequence

$$
\cdots \rightarrow \pi_{i}\left(f^{-1}(\Delta)\right) \rightarrow \pi_{i}(N) \stackrel{\left(p_{1} f\right)_{*}-\left(p_{2} f\right)_{*}}{\longrightarrow} \pi_{i}(M) \rightarrow \pi_{i-1}\left(f^{-1}(\Delta)\right) \rightarrow \cdots .
$$

(B4) For $i \leq n-m-k+1$ there is a natural isomorphism $\pi_{i}\left(N_{1}, f^{-1}(\Delta)\right) \rightarrow$ $\pi_{i}\left(M, N_{2}\right)$ and a surjection for $i=n-m-k+2$, where $\pi_{i}\left(N_{1}, f^{-1}(\Delta)\right)$ is understood as the $i$-th homotopy group of the composition map $f^{-1}(\Delta) \subset N \stackrel{p_{1}}{\rightarrow} N_{1}$.

Some comments on Theorem B are in order.

First, (B1) immediately implies the following intersection theorem, which reduces to the Frankel theorem if $M=\mathbb{P}^{m}$.

Theorem 0.1. Let $M$ be a closed Kähler m-manifold of positive holomorphic $k$ Ricci curvature. If two closed immersed complex submanifolds $f_{i}: N_{i} \rightarrow M, i=$ 1,2 , satisfy $\operatorname{dim}\left(N_{1}\right)+\operatorname{dim}\left(N_{2}\right) \geq m+k-1$, then $f_{1}\left(N_{1}\right) \cap f_{2}\left(N_{2}\right) \neq \emptyset$.

Second, (B2) implies the following regularity result.

Theorem 0.2 (Regularity). Let $M$ be a simply connected closed Kähler m-manifold of positive holomorphic $k$-Ricci curvature. Let $f: N \rightarrow M$ be a complex immersion of a closed complex manifold of dimension $n$. Then $f$ is an embedding, provided $2 n \geq m+k$.

Theorem 0.2 reduces to the Fulton-Hansen regularity theorem $([\underline{\mathrm{FH}}])$ for local complete intersections, which asserts that a finite unramified morphism from an algebraic variety of dimension $n$ to $\mathbb{P}^{m}$ must be an embedding, if the dimension satisfies $2 n>m$.

A Hermitian Kähler symmetric space of compact type has nonnegative bisectional curvature. By the calculation in $[\mathrm{KW}]$ on complex positivity together with Theorem 0.2 we get

Corollary 0.3. Let $M$ be a Kähler manifold and $N$ a closed complex manifold of dimension $n$. Then any complex immersion $f: N \rightarrow M$ is an embedding if

(i) $M=G r_{p}\left(\mathbb{C}^{p+q}\right)$, the complex Grassmannian of complex p-planes in $\mathbb{C}^{p+q}$, and $2 n \geq 2 p q-p-q+2$.

(ii) $\bar{M}=G r_{2}\left(\mathbb{R}^{p+2}\right)$, the Grassmannian of oriented real 2-planes in $\mathbb{R}^{p+2}$ (i.e. the complex quadric), and $2 n \geq p+2$. 
Third, (B3) is a generalization of Deligne's connectedness theorem in $\mathbb{P}^{m}$ (cf. [GM], page 27).

Fourthly, (B4) easily implies the following

Theorem 0.4. Let $M$ be a closed Kähler m-manifold of positive holomorphic $k$ Ricci curvature. Let $N_{1}, N_{2}$ be embedded closed complex submanifolds in $M$ of dimensions $n_{1}, n_{2}$, respectively. Set $n=n_{1}+n_{2}$. Then there is a natural isomorphism,

$$
\pi_{i}\left(N_{1}, N_{1} \cap N_{2}\right) \rightarrow \pi_{i}\left(M, N_{2}\right),
$$

for $i \leq n-m-k+1$ and a surjection for $i=n-m-k+2$.

Theorem 0.4 reduces to the classical Lefschetz hyperplane section theorem and the Barth theorem when $k=1$, by the Mori-Siu-Yau Theorem ([SY], [Mo]).

Finally, (B2) implies

Theorem 0.5. Let $M$ be a simply connected closed Kähler m-manifold of positive holomorphic $k$-Ricci curvature. Let $g: N \rightarrow M$ be a closed complex immersion of codimension d. Let $f: X \rightarrow M$ be another closed complex immersion of dimension $i$. Then $f^{-1}(g(N))$ is connected if $i \geq d+k$.

Note that Theorem 0.5 may be considered as a Bertini-type theorem which asserts the same conclusion if replacing $M$ by $\mathbb{P}^{m}([\mathrm{Fu}])$.

Remark 0.6. In all results of this paper the ambient manifold $M$ could be relaxed to be complete. The reason is that Theorem 5.1 of the fifth section, together with the dimension assumptions in our results, imply the compactness of the ambient manifold.

Remark 0.7. Theorems A and B may hold under some partially positive curvature conditions (e.g. almost everywhere positive curvature). For instance, Theorem 0.2 and Corollary 0.3 hold if $M$ has positive holomorphic $k$-Ricci curvature outside $N$ or if $M$ has positive holomorphic $k$-Ricci curvature at points in $N$.

Remark 0.8. For a holomorphic vector bundle $E \rightarrow M$, Griffiths proved that the ampleness of $E$ implies the so-called Griffiths positivity [G]. A generalized Lefschetztype theorem holds for the zero locus of a holomorphic section of any holomorphic vector bundle $E \rightarrow M$ satisfying the Griffiths positivity. Sommese $\underline{\text { So }}$ has defined the notion of $k$-ample for a bundle, 0 -ample being ample, and $k$ a sort of defect from ampleness. We do not know if the positivity of the holomorphic $k$-Ricci curvature implies the $(k-1)$-ampleness of the canonical bundle $K \rightarrow M$ for $k>1$.

We conclude this section with two more remarks extending our theorems to certain more general settings.

Remark 0.9. A Hermitian manifold $M$ with complex structure $J$ and Hermitian metric $g$ is called locally conformal Kähler (l.c. Kähler, in brief, cf. [Or]), if around every point there exists a coordinate chart on which $g$ is conformal to a Kähler metric. It is well known that on a l.c.K manifold there exists a globally defined closed 1-form $\omega$ (the Lee form) such that $d \Omega=\omega \wedge \Omega$, where $\Omega$ is the Kähler form of $M$. The contravariant vector field $\omega^{\#}$ of $\omega$ is called the Lee vector field. Theorems A and B hold identically if $M$ is a l.c. Kähler manifold, and $f: N \rightarrow M \times M$ is a complex immersion tangent to the Lee vector field $\omega^{\#}$ (compare $[\mathrm{Or}$ ] for the Frankel type theorem). 
Remark 0.10. An almost Hermitian manifold $M$ with almost complex structure $J$ is called nearly Kähler if the Levi-Civita connection of the Hermitian metric satisfies $\nabla_{X}(J) X=0$ for any vector field $X$ (cf. $[\mathrm{Gr}$ ). Theorems A and B hold identically for nearly Kähler manifolds (compare [Gr] section 10 for a Frankel type theorem).

This paper was partially inspired by the works [FMR], where a connectedness principle was developed for totally geodesic submanifolds in the geometry of positive curvature, and [SW], where Morse theory on path spaces are used to obtain theorems of Barth-Lefschetz type.

The rest of the paper is organized as follows:

In Section 1, we apply Morse theory to suitable path spaces.

In Section 2, we prove some indices theorems for energy functions.

In Section 3, we prove Theorems A-B.

In Section 4, we prove Theorems 0.2, 0.4, 0.5 and Corollary 0.3.

\section{Morse theory on PATH SPACES}

Let $M$ be a complete Riemannian manifold without boundary and let $f: N \rightarrow$ $M \times M$ be an immersed complete submanifold of dimension $n$.

A piecewise smooth path in $M(\bmod f)$ is a pair $(x, \gamma)$, where $x \in N$ and a map $\gamma:[0,1] \rightarrow M$ such that:

(i) there is a subdivision $0=t_{0}<t_{1}<\cdots<t_{k}=1$ of $[0,1]$ such that each $\left.\gamma\right|_{\left[t_{i-1}, t_{i}\right]}$ is smooth.

(ii) $(\gamma(0), \gamma(1))=f(x)$.

The set of all piecewise smooth paths $(\bmod f)$ is denoted by $P(M ; f)$.

The topology of $P(M ; f)$ is taken the induced topology from $N \times P(M)$, where $P(M)$ is the space of piecewise smooth paths with the metric topology given by

$$
d\left(\gamma_{0}, \gamma_{1}\right)=\left\{\int_{0}^{1}\left(\left|\dot{\gamma}_{0}(t)\right|-\left|\dot{\gamma}_{1}(t)\right|\right)^{2} d t\right\}^{\frac{1}{2}}+\max _{0 \leq t \leq 1} d_{M}\left(\gamma_{0}(t), \gamma_{1}(t)\right) .
$$

Note that the integral is well defined although $\dot{\gamma}_{i}(i=1,2)$ may not be defined at finitely many points in $[0,1]$.

On the space $P(M ; f)$ there is an energy function $E: P(M ; f) \rightarrow \mathbb{R}$ given by

$$
E(x, \gamma)=\int_{0}^{1}|\dot{\gamma}(t)|^{2} d t
$$

We want to study the topology of $P(M ; f)$ using Morse theory for the function $E$. When $f$ is an embedding, path space with this general boundary condition was studied in [GR] using Morse theory on Hilbert manifolds. Instead, we will use the finite-dimensional approximation methods to reduce to Morse theory in finite dimension.

The tangent space of $P(M ; f)$ at $(x, \gamma)$ is defined as the vector space of piecewise smooth vector fields $W$ along $\gamma$ such that $(W(0), W(1)) \in f_{*}\left(T_{x} N\right)$.

By a standard calculation the first variation of $E$ in the direction $W \in T_{\gamma} P(M, f)$, is given by

$$
\begin{aligned}
\frac{1}{2} E_{*}(W)= & -\int_{0}^{1}\left\langle W(t), \frac{D}{d t} \dot{\gamma}(t)\right\rangle d t \\
& +\langle W(1), \dot{\gamma}(1)\rangle-\langle W(0), \dot{\gamma}(0)\rangle+\sum_{i=1}^{k-1}\left\langle W\left(t_{i}\right), \dot{\gamma}_{-}\left(t_{i}\right)-\dot{\gamma}_{+}\left(t_{i}\right)\right\rangle,
\end{aligned}
$$


where $\dot{\gamma}_{-}$is the left derivative and $\dot{\gamma}_{+}$is the right derivative of $\gamma$, and $\frac{D}{d t}$ is the covariant derivative along $\gamma$. So if $(x, \gamma)$ is a critical point of $E$, then:

(i) $\gamma$ is a smooth geodesic;

(ii) $(\dot{\gamma}(0),-\dot{\gamma}(1))$ is perpendicular to $f_{*}\left(T_{x}(N)\right)$ at $f(x)=(\gamma(0), \gamma(1))$.

Let $W_{1}, W_{2} \in T_{\gamma} P(M ; f)$. If $\gamma$ is a critical point of $E$ we consider any variation $h(t, s, u)$ of $\gamma$ with $\frac{\partial h}{\partial s}(t, 0,0)=W_{1}(t), \frac{\partial h}{\partial u}(t, 0,0)=W_{2}(t)$. Then the second variation of $E$ along $\gamma$, denoted by $E_{* *}\left(W_{1}, W_{2}\right)$, is as follows:

$$
\begin{aligned}
\frac{1}{2} E_{* *}\left(W_{1}, W_{2}\right)= & \int_{0}^{1}\left\{\left\langle\frac{D W_{1}}{d t}, \frac{D W_{2}}{d t}\right\rangle-\left\langle R\left(\dot{\gamma}, W_{1}\right) W_{2}, \dot{\gamma}\right\rangle\right\} \\
& +\left\langle\frac{D}{d s} \frac{\partial h}{d u}(1,0,0), \dot{\gamma}(1)\right\rangle-\left\langle\frac{D}{d s} \frac{\partial h}{d u}(0,0,0), \dot{\gamma}(0)\right\rangle \\
= & \int_{0}^{1}\left\{\left\langle\frac{D W_{1}}{d t}, \frac{D W_{2}}{d t}\right\rangle-\left\langle R\left(\dot{\gamma}, W_{1}\right) W_{2}, \dot{\gamma}\right\rangle\right\} \\
& +\left\langle\alpha\left(\left(W_{1}(0), W_{1}(1)\right),\left(W_{2}(0), W_{2}(1)\right)\right),(-\dot{\gamma}(0), \dot{\gamma}(1))\right\rangle,
\end{aligned}
$$

where $\alpha$ is the second fundamental form of the immersion $f: N \rightarrow M \times M$.

Let $P_{c}(M ; f)=E^{-1}([0, c)) \subset P(M ; f)$. Following Milnor-Morse we define a finite-dimensional approximation to $P_{c}(M ; f)$ as follows:

Choose some subdivision $0=t_{0}<t_{1}<\cdots<t_{k}=1$ of $[0,1]$. Let $B$ be the subspace of $P_{c}(M ; f)$ such that

(i) $f(x)=(\gamma(0), \gamma(1))$;

(ii) $\left.\gamma\right|_{\left[t_{i-1}, t_{i}\right]}$ is a geodesic for each $i=1, \cdots, k$.

Theorem 1.1. Let $M$ be a complete Riemannian manifold, and let $f: N \rightarrow M \times M$ be an immersion where $N$ is a closed Riemannian manifold. Let $c$ be a fixed positive number such that $P_{c}(M ; f)$ is not empty. Then for all sufficiently fine subdivisions $0=t_{0}<t_{1}<\cdots<t_{k}=1$ of $[0,1]$ the set $B$ can be given the structure of a smooth finite-dimensional manifold.

Proof. Mi], Sect. 16.

Let $\left.E\right|_{B}: B \rightarrow \mathbb{R}$ be the restriction of the energy function $E$.

Theorem 1.2. Let $f, N, M$ be as in Theorem 1.1. Then $\left.E\right|_{B}$ is a smooth function. For each $a<c$ the set $\left(\left.E\right|_{B}\right)^{-1}([0, a])$ is compact, and $\left(\left.E\right|_{B}\right)^{-1}([0, a))$ is a deformation retract of the set $P_{a}(M ; f)$. The critical points of $\left.E\right|_{B}$ are precisely the same as the critical points of $E$ in $P_{c}(M ; f)$, that is, the pairs $(x, \gamma)$, where $\gamma$ is a smooth geodesic in $M$ such that $(\dot{\gamma}(0),-\dot{\gamma}(1))$ is normal to $f_{*}\left(T_{x}(N)\right)$ and the energy is less than $c$. The Hessians of $\left.E\right|_{B}$ and $\left.E\right|_{P_{c}(M ; f)}$ have the same index at each critical point $(x, \gamma)$.

Proof. [Mi], Sect. 14 and Sect. 16.

Lemma 1.3. Suppose that every nontrivial critical point $(x, \gamma)$ of $E$ has positive index. Then $f^{-1}(\Delta)$ is not empty.

Proof. Suppose $f^{-1}(\Delta)=\emptyset$. Writing $f(x)=\left(f_{1}(x), f_{2}(x)\right)$, from the compactness of $N$ we conclude that there exists $x \in N$ such that $d\left(f_{1}(x), f_{2}(x)\right)=\delta$ is a positive minimum. In particular $P(M ; f)$ contains no constant path and the energy function on $P(M ; f)$ assumes a positive minimum $\delta^{2}$ at $(x, \gamma)$, where $\gamma$ is a minimal geodesic 
joining $f_{1}(x)$ and $f_{2}(x)$. For this minimal critical point $(x, \gamma)$ the index is clearly zero. A contradiction to the assumption.

We also need the following lemma of Milnor for finite-dimensional manifolds.

Lemma 1.4. Let $X$ be a finite-dimensional smooth manifold and let $f: X \rightarrow \mathbb{R}$ be a real function with minimal value zero. Suppose that for any a the sublevel set $X_{\leq a}=f^{-1}([0, a])$ is compact. Assume that the set $X_{0}$ of minimal points has a neighborhood $U$ with a deformation retraction $r: U \rightarrow X_{0}$, and that all nontrivial critical points have indices greater than $\lambda_{0} \geq 0$. Then $X$ has the homotopy type of a $C W$-complex by attaching cells of dimensions at least $\lambda_{0}+1$ to $X_{0}$. In particular, $\pi_{j}\left(X, X_{0}\right)=0$ for $0 \leq j \leq \lambda_{0}$.

Proof. [Mi], Sect. 22.

Theorem 1.5. Let $M$ be a complete Kähler manifold and let $\Delta$ be the diagonal of $M \times M$. Suppose that $f: N \rightarrow M \times M$ is a complex immersion, where $N$ is a closed manifold. Let $P_{0}=f^{-1}(\Delta)$. If every nontrivial critical point of $E$ on $P(M ; f)$ has index $\lambda>\lambda_{0} \geq 0$, then $P(M ; f)$ has the homotopy type of a $C W$-complex obtained by attaching cells of dimensions at least $\lambda_{0}+1$ to $P_{0}$. In particular, the relative homotopy groups $\pi_{j}\left(P(M ; f), P_{0}\right)=0$ for $0 \leq j \leq \lambda_{0}$.

Proof. Note that each point $x$ in $P_{0}$ could be associated with a constant path at $p_{1} f(x)\left(=p_{2} f(x)\right)$. So $P_{0}$ can be identified with the constant geodesics in $P(M ; f)$. It suffices to prove that for any large value $c, P_{c}(M, f)$ has the homotopy type of a CW-complex obtained by attaching cells of dimensions at least $\lambda_{0}+1$ to $P_{0}$. By Theorem $1.2 P_{c}(M, f)$ deformation retracts to $B$. Moreover, the index for every nontrivial critical point for the restricted energy function is greater than $\lambda_{0}$, too. The space $P_{0}$ is clearly inside $B$. To apply Lemma 1.4 it suffices to prove that there is a neighborhood $U \subset B$ of $P_{0}$ and a retraction $r: U \rightarrow P_{0}$.

Since $f$ is complex analytic with respect to holomorphic coordinates in $N$ and $M \times M$, the minimal set $P_{0}$ (resp. $B$ ) may be identified with an analytic subvariety $\left\{(x, f(x), \cdots, f(x)): x \in f^{-1}(\Delta)\right\}$ (resp. an open subvariety of $N \times(M \times M) \times$ $\cdots \times(M \times M))$. It is well known that any analytic subvariety is triangulable, as a subcomplex of $N \times(M \times M) \times \cdots \times(M \times M)$. Therefore an open regular neighborhood of this subcomplex is a desired open neighborhood $U$. The desired result follows.

\section{INDICES THEOREMS FOR CRITICAL POINTS OF ENERGY FUNCTIONS}

We start with two elementary lemmas in linear algebra.

Lemma 2.1. Let $V$ be a j-dimensional real linear space with an inner product $\langle.,$.$\rangle .$ Let $I(.,$.$) be a symmetric bilinear form on V. Fix 1 \leq k \leq j$. Assume that for all orthonormal vectors $e_{1}, \cdots, e_{k}$ we have

$$
\sum_{i=1}^{k} I\left(e_{i}, e_{i}\right)<0 .
$$

Then the index $\lambda$ of I satisfies $\lambda \geq j-k+1$.

Proof. It follows from the proof of (2.1.1) in Lemma 2.1 of [FMR]. 
Lemma 2.2. Let $V$ be a $2 j$-dimensional real linear space with inner product $\langle.,$. and an isomorphism $J: V \rightarrow V$. Let $I(.,$.$) be a real symmetric bilinear form on$ $V$. Assume that for all nonzero vector $X \in V$ it holds that

$$
I(X, X)+I(J X, J X)<0 .
$$

Then the index $\lambda$ of I satisfies $\lambda \geq j$.

Proof. Consider an orthonormal basis $\beta$ of eigenvectors of the symmetric linear map $A: V \rightarrow V$, where $\langle A v, w\rangle=I(v, w)$. Let $W$ be the space generated by the vectors $E \in \beta$ with $I(E, E) \geq 0$. Note that $A$ is nonnegative on $W$ and that the index of $I$ is equal to $2 j-\operatorname{dim}(W)$. So it suffices to prove that $\operatorname{dim}(W) \leq j$. Suppose not; then the intersection $J W \cap W$ is a nontrivial linear subspace. For any nontrivial $w=J v \in W \cap J W$, where $v \in W$, we have $I(v, v) \geq 0$ and also $I(w, w) \geq 0$. Thus $I(v, v)+I(J v, J v) \geq 0$. A contradiction. The desired result follows.

Let $M$ be a complete Kähler $m$-manifold. Let $f: N \rightarrow M \times M$ be an immersed closed complex submanifold. Let $W_{1}, W_{2} \in T_{(x, \gamma)} P(M ; f)$ be tangent vectors at $(x, \gamma)$. Let $\alpha$ be the second fundamental form of $f$ in $M \times M$. Recall that the second variation of the energy function $E$ along a critical point $(x, \gamma)$ reads

$$
\begin{aligned}
\frac{1}{2} E_{* *}\left(W_{1}, W_{2}\right)=\int_{0}^{1} & \left\{\left\langle\frac{D W_{1}}{d t}, \frac{D W_{2}}{d t}\right\rangle-\left\langle R\left(\dot{\gamma}, W_{1}\right) W_{2}, \dot{\gamma}\right\rangle\right\} \\
+ & \left\langle\alpha\left(\left(W_{1}(0), W_{1}(1)\right),\left(W_{2}(0), W_{2}(1)\right)\right),(-\dot{\gamma}(0), \dot{\gamma}(1))\right\rangle .
\end{aligned}
$$

Now let $W$ be parallel along $\gamma$. Set

$$
\mathcal{W}=(W(0), W(1)) \text { and } \eta=(-\dot{\gamma}(0), \dot{\gamma}(1)) .
$$

Note that $\nabla_{\mathcal{W}} \mathcal{W}=-\nabla_{J \mathcal{W}} J \mathcal{W}$ in the Kähler manifold $M \times M$. Therefore, in the sum $\frac{1}{2} E_{* *}(W, W)+\frac{1}{2} E_{* *}(J W, J W)$ the boundary term equals

$$
\left\langle\nabla_{\mathcal{W}} \mathcal{W}, \eta\right\rangle+\left\langle\nabla_{J \mathcal{W}} J \mathcal{W}, \eta\right\rangle=0
$$

Therefore

$$
\frac{1}{2} E_{* *}(W, W)+\frac{1}{2} E_{* *}(J W, J W)=\int_{0}^{1}-\{\langle R(\dot{\gamma}, W) W, \dot{\gamma}\rangle+\langle R(\dot{\gamma}, J W) J W, \dot{\gamma}\rangle\} .
$$

If $W$ is a parallel vector field along $\gamma$ with $\mathcal{W}=(W(0), W(1)) \in f_{*}\left(T_{x} N\right)$, then $J \mathcal{W}=(J W(0), J W(1))$ is also tangent to $N$ at $x$, since $f$ is a complex immersion. Let $\mathcal{V}$ be the real linear space spanned by vectors $(W(0), W(1))$, where $W$ is a parallel vector field along $\gamma$ with $W$ orthogonal to $\dot{\gamma}$ and $J \dot{\gamma}$. The complex dimension of $\mathcal{V}$ is $m-1$. If $\mathcal{W}=(W(0), W(1)) \in \mathcal{V}$, then $J \mathcal{W} \in \mathcal{V}$ and both $\mathcal{W}, J \mathcal{W}$ are orthogonal to $\eta=(-\dot{\gamma}(0), \dot{\gamma}(1))$ and $J \eta$. We have the complex dimension

$$
\operatorname{dim}_{\mathbb{C}}\left(\mathcal{V} \cap f_{*}\left(T_{x} N\right)\right) \geq m-1+n-(2 m-1)=n-m .
$$

Recall that if $\mathcal{W} \in \mathcal{V} \cap f_{*}\left(T_{x} N\right)$, then $W$ is a tangent vector of $P(M, f)$ at $(x, \gamma)$.

Theorem 2.3. Let $M$ be a complete Kähler $m$-manifold of positive holomorphic $k$ Ricci curvature. Let $f: N \rightarrow M \times M$ be a complex immersion of a closed manifold $N$ of dimension $n$. If $(x, \gamma)$ is a nontrivial critical point (i.e. $\gamma$ is not a constant geodesic) for the energy function $E$ on $P(M, f)$ then the index $\lambda$ of the critical point $(x, \gamma)$ satisfies

$$
\lambda \geq n-m-k+1
$$


Proof. We can assume that $k \leq n-m$ since otherwise there is nothing to prove.

Let $V \subset T_{(x, \gamma)} P(M, f)$ be the complex vector space spanned by parallel vector fields $W$ along $\gamma$ orthogonal to the complex line $\dot{\gamma} \wedge J \dot{\gamma}$ such that $(W(0), W(1)) \in \mathcal{V} \cap$ $f_{*}\left(T_{x} N\right)$. Note that $V$ and $\mathcal{V} \cap f_{*}\left(T_{x} N\right)$ are $\mathbb{C}$-isomorphic, hence $\operatorname{dim}_{\mathbb{C}}(V) \geq n-m$.

Consider the real symmetric bilinear form

$$
I(v, w)=E_{* *}(v, w)+E_{* *}(J v, J w)
$$

on $V$ and the real inner product

$$
\langle X, Y\rangle:=\langle X(0), Y(0)\rangle .
$$

Note that the index of $E_{* *}$ is not less than the index of $E_{* *}$ restricted to $V$. Therefore by Lemma 2.2 it suffices to prove that there is a complex subspace $V_{0} \subset V$ of dimension at least $n-m-k+1$, such that for any nontrivial vector $X \in V_{0}$ it holds that

$$
I(X, X)=E_{* *}(X, X)+E_{* *}(J X, J X)<0,
$$

where $J$ is the complex structure on $V_{0}$.

Consider $V$ as a real vector space. Let $B: V \rightarrow V$ be the real symmetric linear map defined by $\langle B v, w\rangle=I(v, w)$. Since $I(X, X)=I(J X, J X)$, it is easy to see that $J B=B J$. So $B$ is also a $\mathbb{C}$-linear map. Since $B$ is symmetric, its eigenvalues are real, hence there is a basis of $V$ of orthonormal eigenvectors $e_{1}, J e_{1}, \cdots, e_{j}, J e_{j}$. Thus there exists an orthogonal decomposition $V=\mathbb{R}^{j} \oplus J \mathbb{R}^{j}$ such that $\mathbb{R}^{j}$ and $J \mathbb{R}^{j}$ are $B$-invariant. Let $C: \mathbb{R}^{j} \rightarrow \mathbb{R}^{j}$ be the restriction of $B$. Note that $B=C \oplus C$, with respect to the above basis.

Take any $k$ orthonormal vectors $W_{1}, \cdots, W_{k}$ in $\mathbb{R}^{j}$. Since $W_{1}, J W_{1}, \cdots, W_{k}, J W_{k}$ are orthonormal, the holomorphic $k$-Ricci curvature

$$
\operatorname{Ric}_{k}^{H}\left(\dot{\gamma} \wedge J \dot{\gamma}, W_{1} \wedge J W_{1}, \cdots, W_{k} \wedge J W_{k}\right)
$$

is well defined. So we have

$$
\begin{aligned}
\sum_{i=1}^{k} I\left(W_{i}, W_{i}\right) & =\sum_{i=1}^{k}\left(E_{* *}\left(W_{i}, W_{i}\right)+E_{* *}\left(J W_{i}, J W_{i}\right)\right) \\
& =\sum_{i=1}^{k} \int_{0}^{1}-\left\{\left\langle R\left(\dot{\gamma}, W_{i}\right) W_{i}, \dot{\gamma}\right\rangle+\left\langle R\left(\dot{\gamma}, J W_{i}\right) J W_{i}, \dot{\gamma}\right\rangle\right\} \\
& =\int_{0}^{1}\left\{-\operatorname{Ric}_{k}^{H}\left(\dot{\gamma} \wedge J \dot{\gamma}, W_{1} \wedge J W_{1}, \cdots, W_{k} \wedge J W_{k}\right)\right\} \\
& <0 .
\end{aligned}
$$

Thus we conclude by Lemma 2.1 that the index $\mu$ of $I$ restricted to $\mathbb{R}^{j}$ satisfies

$$
\mu \geq j-k+1 \geq n-m-k+1 .
$$

Therefore there exists an $(n-m-k+1)$-dimensional real vector subspace of $\mathbb{R}^{j}$ spanned by orthonormal eigenvectors of $B$, say $e_{1}, \cdots, e_{n-m-k+1}$ for convenience, such that $I$ is negative definite on $\operatorname{Span}\left\{e_{1}, \cdots, e_{n-m-k+1}\right\}$. Recall that $I\left(J e_{i}, J e_{i}\right)=I\left(e_{i}, e_{i}\right)<0$. Hence $I$ is negative definite on the real linear subspace

$$
V_{0}=\operatorname{Span}\left\{e_{1}, J e_{1}, \cdots, e_{n-m-k+1}, J e_{n-m-k+1}\right\} .
$$

The desired result follows. 
The inequality in Theorem 2.3 may be improved when $N$ is the Riemannian product $N_{1} \times N_{2}$, where $N_{1}$ and $N_{2}$ are complex immersed submanifolds in $M$.

Theorem 2.4. Let $M$ and $f: N \rightarrow M \times M$ be as in Theorem 2.3. Let $N=N_{1} \times N_{2}$ and $f=\left(f_{1}, f_{2}\right)$ where $f_{i}: N_{i} \rightarrow M, i=1,2$, are complex immersions of closed manifolds. If $(x, \gamma)$ is a nontrivial critical point for the energy function $E$ on $P(M, f)$, then the index $\lambda$ of $E_{* *}$ at $(x, \gamma)$ satisfies

$$
\lambda \geq n-m-k+2 \text {. }
$$

Proof. We can assume that $k \leq n-m+1$. Let $\mathcal{V}$ be as in the proof of Theorem 2.3. If $x=\left(x_{1}, x_{2}\right)$, it is easy to see that the critical point $(x, \gamma)$ satisfies $\dot{\gamma}(0) \perp\left(f_{1}\right)_{*}\left(T_{x_{1}} N_{1}\right)$ and $\dot{\gamma}(1) \perp\left(f_{2}\right)_{*}\left(T_{x_{2}} N_{2}\right)$. Therefore $(\dot{\gamma}(0), 0)$ and $(J \dot{\gamma}(0), 0)$ are both orthogonal to $\left(f_{1}\right)_{*}\left(T_{x_{1}} N_{1}\right) \times\left(f_{2}\right)_{*}\left(T_{x_{2}} N_{2}\right)=f_{*}\left(T_{x} N\right)$; similarly $(0, \dot{\gamma}(1))$ and $(0, J \dot{\gamma}(1))$ are both orthogonal to $f_{*}\left(T_{x} N\right)$. Obviously, all the four vectors $(\dot{\gamma}(0), 0),(J \dot{\gamma}(0), 0),(0, \dot{\gamma}(1))$ and $(0, J \dot{\gamma}(1))$ are orthogonal to $\mathcal{V}$. Therefore the intersection $f_{*}\left(T_{x} N\right) \cap \mathcal{V}$ has

$$
\operatorname{dim}_{\mathbb{C}}\left(\mathcal{V} \cap f_{*}\left(T_{x} N\right)\right) \geq n-m-k+2 .
$$

For the same reasoning as above it follows that the index $\lambda$ of $E_{* *}$ at the critical point $(x, \gamma)$ satisfies $\lambda \geq n-m-k+2$.

\section{Proofs of Theorems A-B}

Let $M$ be a complete Kähler manifold. Let $P(M)$ denote the space of $C^{k}$ paths $\gamma:[0,1] \rightarrow M$. Let $\pi: P(M) \rightarrow M \times M$ be the projection to the pair of end points (i.e. $(\gamma(0), \gamma(1)))$. It is a standard result in topology that $\pi$ is a Serre fibration

$$
\Omega M \rightarrow P(M) \stackrel{\pi}{\longrightarrow} M \times M
$$

with fiber the loop space $\Omega M$ with a fixed base point. By definition we know that the projection to the first factor $p: P(M, f) \rightarrow N$ is exactly the pullback fibration by $f: N \rightarrow M \times M$ from $\pi: P(M) \rightarrow M \times M$. In particular, there is a homotopy exact sequence

$$
\cdots \rightarrow \pi_{i}(P(M ; f)) \rightarrow \pi_{i}(N) \rightarrow \pi_{i-1}(\Omega M) \rightarrow \pi_{i-1}(P(M ; f)) \rightarrow \cdots .
$$

Proof of Theorem A. By Lemma 1.3 and Theorem 2.3, (A1) follows.

Note that $\pi_{i-1}(\Omega M)=\pi_{i}(M)$. In particular, $\Omega M$ is path connected if $M$ is simply connected. Then $P(M ; f)$ is path connected. If $n>m+k$, by Theorem 1.5 and the index Theorem $2.3, P(M ; f)$ has the homotopy type of a CW-complex, obtained from $f^{-1}(\Delta)$ by attaching cells of dimensions at least 2 . Thus $f^{-1}(\Delta)$ is path connected. This proves (A2).

By Theorem 1.5 and Theorem 2.3 we know that $\pi_{i}\left(P(M ; f) ; f^{-1}(\Delta)\right)=0$ if $i \leq n-m-k$. By the above homotopy exact sequence we get the desired exact sequence. It is easy to see that the homomorphism from $\pi_{i}(N) \rightarrow \pi_{i}(M)$ is given by $\left(p_{1} f\right)_{*}-\left(p_{2} f\right)_{*}$, where $p_{1}, p_{2}: M \times M \rightarrow M$ are the projections to the first and the second factor, respectively (cf. GR]). This implies (A3).

Notation. If $f: Y \rightarrow X$ is a map, we use $\pi_{i}(X, Y)$ to denote the homotopy group $\pi_{i}\left(X_{f}, Y\right)$, where $X_{f}=X \cup_{f} Y \times[0,1]$ is the mapping cylinder of $f$. 
Proof of Theorem B. The proofs of (B1)-(B3) are the same as in the proof of Theorem A.

To prove (B4) instead of deriving from (B3) we use more geometric methods.

Let $p_{1}: P(M ; f) \rightarrow N_{1}$ denote the composition of the bundle projection $p$ with the projection from $N_{1} \times N_{2} \rightarrow N_{1}$ to the first factor. Note that $p_{1}$ is a fibration with fiber $V$, the pullback fibration fitting in the commutative diagram

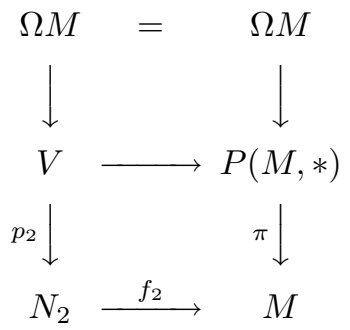

where the right side is the principal path fibration. Note that $P(M, *)$ is contractible. By the above diagram $V$ is the homotopy fiber of the map $f_{2}: N_{2} \rightarrow M$. Therefore by the fibration homotopy exact sequence it follows that

$$
\pi_{i}(V) \approx \pi_{i+1}\left(M, N_{2}\right)
$$

for all $i$. This together with the homotopy exact sequence for the fibration

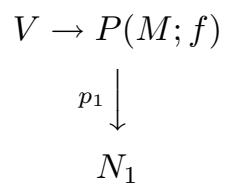

and the long exact sequence for the map $i_{1}: f^{-1}(\Delta) \rightarrow N_{1}$ gives a commutative diagram:

$$
\begin{aligned}
& \pi_{i+1}\left(N_{1}\right) \rightarrow \pi_{i+1}\left(N_{1}, f^{-1}(\Delta)\right) \rightarrow \pi_{i}\left(f^{-1}(\Delta)\right) \rightarrow \pi_{i}\left(N_{1}\right) \rightarrow \pi_{i}\left(N_{1}, f^{-1}(\Delta)\right) \\
& =\downarrow \quad\left(f_{1}\right)_{*} \downarrow \quad \operatorname{surj} \downarrow \quad=\downarrow \quad\left(f_{1}\right)_{*} \downarrow \\
& \pi_{i+1}\left(N_{1}\right) \rightarrow \quad \pi_{i+1}\left(M, N_{2}\right) \quad \rightarrow \pi_{i}(P(M ; f)) \rightarrow \pi_{i}\left(N_{1}\right) \rightarrow \quad \pi_{i}\left(M, N_{2}\right)
\end{aligned}
$$

The middle homomorphism is surjective for $i \leq n-m-k+1$ since by Theorem 1.5 and Theorem 2.4, $\pi_{i}\left(P(M ; f), f^{-1}(\Delta)\right)=0$. By the 5-lemma the commutative diagram above implies that

$$
\left(f_{1}\right)_{*}: \pi_{i}\left(N_{1}, f^{-1}(\Delta)\right) \rightarrow \pi_{i}\left(M, N_{2}\right)
$$

is an isomorphism for all $i \leq n-m-k+1$ and a surjection for $i=n-m-k+2$. The desired result follows.

\section{Proofs of Theorems $0.2,0.4,0.5$ and Corollary 0.3}

Proof of Theorem 0.2. Since $f: N \rightarrow M$ is an immersion, it suffices to show that $f$ is a one-to-one map. Note that $(f, f)^{-1}(\Delta)=\{(x, x), x \in N\} \cup\{(x, y): f(x)=$ $f(y), x \neq y)\}$. Hence, if $f$ is not one-to-one, then $f^{-1}(\Delta)$ is not connected; a contradiction to (B2).

Proof of Corollary 0.3. Let $l$ be the complex positivity of $M$. Observe that $k=$ $m-l+1$. The desired result follows by Theorem 0.2 and the calculation of $l$ in KW. 
Proof of Theorem 0.4. In (B4), consider $f_{1}=i_{1}: N_{1} \subset M$ and $f_{2}=i_{2}: N_{2} \subset M$. It is clear that $f^{-1}(\Delta)=N_{1} \cap N_{2}$. The desired result follows.

Proof of Theorem 0.5. Consider the totally geodesic immersion $(f, g): X \times N \rightarrow$ $M \times M$. By (B2), $(f, g)^{-1}(\Delta)$ is connected and thus $f^{-1}(g(N))=p_{1}\left((f, g)^{-1}(\Delta)\right)$ is connected.

\section{Compactness of the Ambient manifold}

By using standard arguments on Ricatti equations (see [MZ, Gu]) we can obtain the following result.

Theorem 5.1. Let $M$ be a complete and noncompact m-dimensional Kähler manifold of positive holomorphic $k$-Ricci curvature. Then $M$ does not admit complex immersions of closed manifolds $N$ of dimension $n \geq k$.

Proof. Assume by contradiction that $M$ is noncompact and $f: N \rightarrow M$ is a closed complex immersion with $\operatorname{dim} N=n \geq k$. Since $N$ is compact there exists a ray $\gamma:[0,+\infty) \rightarrow M$ with $\gamma(0)=f(x), x \in N$ and $t=d(\gamma(t), f(N))$ for all $t$. Consider orthonormal vectors $e_{1}, J e_{1}, \cdots, e_{k}, J e_{k} \in f_{*}\left(T_{x} N\right)$. Consider the parallel transports $e_{i}(t), J e_{i}(t)$ along $\gamma$. Set

$$
\varphi(t)=\frac{1}{2 k} \operatorname{Ric}_{k}^{H}\left(\dot{\gamma}(t) \wedge J \dot{\gamma}(t), e_{1}(t) \wedge J e_{1}(t), \cdots, e_{k}(t) \wedge J e_{k}(t)\right) .
$$

Let $g$ be the solution of the equation

$$
\left\{\begin{array}{l}
g^{\prime \prime}+\varphi g=0 \\
g(0)=1, \quad g^{\prime}(0)=0 .
\end{array}\right.
$$

First we assume that $g$ is positive on $(0, b)$ and $g(b)=0$. Consider fields $X_{i}(t)=$ $g(t) e_{i}(t)$, for $t \in[0, b]$. Since $\gamma$ is minimizing we have

$$
0<\frac{1}{2} \sum_{i=1}^{k}\left(E_{* *}\left(X_{i}, X_{i}\right)+E_{* *}\left(J X_{i}, J X_{i}\right)\right) .
$$

By the other hand the second variation formula implies that

$$
\begin{aligned}
\frac{1}{2} I\left(X_{i}, X_{i}\right): & =\frac{1}{2}\left(E_{* *}\left(X_{i}, X_{i}\right)+E_{* *}\left(J X_{i}, J X_{i}\right)\right) \\
& =\int_{0}^{b}\left\{\left|\frac{D X_{i}}{d t}\right|^{2}+\left|\frac{D J X_{i}}{d t}\right|^{2}-\left(\left\langle R\left(\dot{\gamma}, X_{i}\right) X_{i}, \dot{\gamma}\right\rangle+\left\langle R\left(\dot{\gamma}, J X_{i}\right) J X_{i}, \dot{\gamma}\right\rangle\right)\right\},
\end{aligned}
$$

where we used that $\nabla_{X_{i}} X_{i}+\nabla_{J X_{i}} J X_{i}=0$, since $f$ is a complex immersion. So we obtain

$$
\begin{aligned}
0<\frac{1}{2} \sum_{i=1}^{k} I\left(X_{i}, X_{i}\right): & =\int_{0}^{b}\left\{2 k g^{\prime 2}-g^{2} \sum_{i=1}^{k}\left(\left\langle R\left(\dot{\gamma}, e_{i}\right) e_{i}, \dot{\gamma}\right\rangle+\left\langle R\left(\dot{\gamma}, J e_{i}\right) J e_{i}, \dot{\gamma}\right\rangle\right)\right\} \\
& =\int_{0}^{b}\left\{2 k g^{\prime 2}-g^{2} \operatorname{Ric}_{k}^{H}\left(\dot{\gamma} \wedge J \dot{\gamma}, e_{1} \wedge J e_{1}, \cdots, e_{k} \wedge J e_{k}\right)\right\} \\
& =\int_{0}^{b}\left\{2 k\left(g^{\prime 2}-g^{2} \varphi\right)\right\}=\int_{0}^{b} 2 k\left(g g^{\prime}\right)^{\prime}=0,
\end{aligned}
$$


since $g$ satisfies (5.1) and $g^{\prime}(0)=g(b)=0$. This contradiction shows that $g$ is positive for all $t \geq 0$. By setting $u=g^{\prime} / g$ we obtain a Ricatti equation

$$
\left\{\begin{array}{l}
u^{\prime}(t)+u^{2}(t)+\varphi(t)=0, \\
u(0)=0
\end{array}\right.
$$

defined for all $t \geq 0$. Thus it follows from [MZ] or from [Gu] that

$$
\liminf _{s \rightarrow+\infty} \int_{0}^{s} \varphi(t) d t \leq 0,
$$

and this contradicts the positivity of the holomorphic $k$-Ricci curvature. Theorem 5.1 is proved.

\section{ACKNOWLEDGMENT}

The first author thanks Universidade Federal Fluminense (UFF) for its hospitality during the preparation of this work.

\section{REFERENCES}

[AF] A. Andteotti; T. Frankel, The Lefschetz theorem on hyperplane sections, Ann. Math 69 (1959), 713-717. MR0177422 (31:1685)

[Ba] W. Barth, Transplating cohomology classes in complex projective space, Amer. J. Math. 92 (1970), 951-967. MR0287032 (44:4239)

[FMR] F. Fang; S. Mendonça; X. Rong, A Connectedness principle in the geometry of positive curvature, preprint, 2002, to appear in Comm. Analysis and Geometry.

[Fr] T. Frankel, Manifolds of positive curvature, Pacific J. Math. 11 (1961), 165-174. MR0123272 (23:A600)

[Fu] W. Fulton, On the topology of algebraic varieties, Proc. Symp. in Pure Math. 46 (1987), 15-46. MR0927947 (89c:14027)

[FH] W. Fulton; J. Hansen, A connectedness theorems for projective varieties, with applications to intersections and singularities of mappings, Ann. Math 110 (1979), 159-166. MR0541334 (82i:14010)

[FL] W. Fulton; R. Lazarsfeld, Connectivity and Its Applications in Algebraic Geometry, Lecture Notes in Mathematics 862, Springer-Verlag, 26-92. MR0644817 (83i:14002)

[GK] S. Goldberg; S. Kobayashi, Holomorphic bisectional curvature, J. Differential. Geom. 1 (1967), 225-233. MR0227901 (37:3485)

[GM] M. Goresky; R. MacPherson, Stratified Morse theory, Springer-Verlag, New York, 1988. MR0932724 (90d:57039)

[Gr] A. Gray, Nearly Kähler manifolds, J. Diff. Geom. 4 (1970), 283-309. MR 0267502 (42:2404)

[G] P. Griffiths, Hermitian differential geometry, Chern classes, and positive vector bundles, Global Analysis, Univ. Tokyo Press (1969), 195-251. MR0258070 (41:2717)

[GR] K. Grove, Geodesics satisfying general boundary conditions, Comment. Math. Helv. (1973), 376-381. MR0438386 (55:11300)

$[\mathrm{Gu}]$ F. F. Guimarães, The integral of the scalar curvature of complete manifolds without conjugate points, J. Differential Geom. 36 (1992), 651-662. MF1189499 (93j:53055)

[KW] M. Kim; J. Wolfson, Theorems of Barth-Lefschetz type on Kähler manifolds of non-negative bisectional curvature, Forum Math. 15 (2003), 261-273. MR1956967 (2004b:32036)

[Le] S. Lefschetz, L'analysis situs et la geometrie algebrique, Gauthier-Villars, Paris (1924). MR0033557 (11:456c)

[Mi] J. Milnor, Morse theory, Ann. Math. Stud. Princeton University Press (1963). MR0163331 (29:634)

[Mo] S. Mori, Projective manifolds with ample tangent bundles, Ann. of Math. (2) 110 (1979), 593-606. MR 0554387 (81j:14010)

[MZ] S. Mendonça; D. Zhou, Curvature conditions for immersions of submanifolds and applications, Compositio Math. 137 (2003), 211-226. MR1985004|(2004c:53093) 
[Ok] C. Okonek, Barth-Lefschetz theorems for singular spaces, J. Reine. Angew Math. 374 (1987), 24-38. MR0876219 (88c:14029)

[Or] L. Ornea, A theorem on non-negatively curved locally conformal Kaehler manifolds, Rendi. di Matematica 12 (1992), 257-262. MR1186159(93h:53071)

[SW] R. Schoen; J. Wolfson, Theorems of Barth-Lefschetz types and Morse theory on the spaces of paths, Math. Zeit. 229 (1998), 77-89. MR.1649314 (2000i:58021)

[Sh] Z. Shen, On complete manifolds of nonnegativee kth-Ricci curvature, Trans of A.M.S. 338 (1993), 289-310. MR1112548 (93j:53054)

[SY] Y.-T. Siu, S.-T. Yau, Compact Kähler manifolds with positive bisectional curvature, Invent. Math. 59 (1980), 189-204. MR0577360 (81h:58029)

[So] A. Sommese, Complex subspaces of homogeneous complex manifolds II- Homotopy Results, Nagoya Math. J. 86 (1982), 101-129. MR0661221 (84d:32040)

[Wu H. Wu, Manifolds of partially positive curvature, Indiana Univ. Math. J 36 (1987), 525548. MF.0905609 (88k:53068)

Nankai Institute of Mathematics, Nankai University, Tianjin 300071, People's RePUBLIC OF CHINA

E-mail address: fuquanfang@eyou.com

Departamento de Análise, Universidade Federal Fluminense (UFF), Niterói, 24020-140 RJ BRAZIL

E-mail address: sxmendonca@hotmail.com

E-mail address: mendonca@mat.uff.br 